\title{
A mechanism for episodic subduction on Venus
}

\author{
A.C. Fowler \\ Mathematical Institute, Oxford University, Oxford, England
}

\section{S.B.G. O'Brien}

Department of Mathematics, University of Limerick, Limerick, Republic of Ireland

\begin{abstract}
We propose a mechanism previously developed as a hypothetical cause of the initiation of subduction in the Earth's mantle, to describe a situation where such subduction may occur transiently, at irregular intervals of time. It has been suggested that tectonics on Venus may be described by such a scenario. In our model, a subduction event is followed by resumption of high Rayleigh number mantle convection below a stagnant lithosphere which thickens due to conductive cooling. As it thickens, differential buoyancy causes large lithospheric stresses which eventually lead to (plastic) failure in the upper portions of the lithosphere. This plastic zone thickens faster than the lithosphere, so that at some critical time, it reaches the base of the lithosphere. At this point, the effective lithosphere viscosity decreases to that of the underlying mantle, and subduction can occur. We suggest that this is mechanistically consistent with the postulated Venusian tectonic style.
\end{abstract}

\section{Introduction}

The tectonic style of Venus is very different from that of Earth [Solomon, 1993]. In particular, the plate tectonics of Earth is apparently absent [Solomon et al., 1992], as evidenced by the random distribution of impact craters over the surface of Venus, indicating an average age of some 500 m.y. [Schaber et al., 1992; Phillips et al., 1992]. Despite the absence of plate tectonic crustal recycling, Venus is a tectonically active planet, with features such as the equatorial highlands and the coronae being associated with uplift due to mantle plumes [Solomon and Head, 1991; Koch, 1994; Kiefer and Hager, 1991]. The coronae, in particular, are often associated with arcuate trenches which resemble, and have the topography of, subduction zones on the Earth [McKenzie et al., 1992; Sandwell and Schubert, 1992]; it has therefore been suggested [Sandwell and Schubert, 1992] that in fact these coronal trenches are indeed incipient subduction zones.

Their role in the tectonics of Venus is then enigmatic. The lack of a permanent plate tectonic cycle on Venus suggests that any subduction which does occur should be transient, and indeed this is consistent with theories of the resurfacing of Venus which have been put forward based on the impact crater distribution. The uniform distribution is most obviously explained by allowing a global resurfacing model (GRM) [Schaber et al., 1992], whereby violent convection in the Venusian mantle some 500 m.y. ago causes global overturn, since which time

Copyright 1996 by the American Geophysical Union.

Paper number 95JE03261.

0148-0227/96/95JE-03261\$05.00 subduction has ceased. More detailed consideration of various resurfacing models by Phillips et al. [1992] led them to conclude that resurfacing was more likely to occur at shorter intervals over small patches of the crust. Nevertheless, Strom et al. [1994] have reasserted the original GRM (terminating about 300 m.y. ago) on the basis of a larger crater sample size, and more elaborate statistical tests. Although not essential to the argument of this paper, we will use the GRM as a paradigm.

In order to explain how a planetary mantle can act in this way, various ideas have been put forward. Herrick and Parmentier [1994] suggested that overturn could occur in a layered mantle convection system due to competition between thermal and compositional buoyancy. Steinbach and Yuen [1992] suggested resurfacing might be attributed to a transition from phase-changeinduced layered convection to whole mantle convection as the planet cooled. Parmentier and Hess [1992] suggested a mechanism associated with evolving compositional and thermal buoyancy due to surface cooling and volcanic fractionation. Arkani-Hamed et al. [1993] associate resurfacing with a transition from oscillatory to steady convection due to planetary cooling and thus a decreasing Rayleigh number.

All of these accounts rely on the properties of convection, without considering the dynamics of subduction. In particular, these studies mostly rely on properties of constant viscosity convection, or where they do not [e.g., Lenardic et al., 1993], the viscosity is constrained between limits. However, one of the most significant facts concerning the rheology of mantle rocks is the sensitivity of their viscosity to temperature [Kirby, 1983]. For olivine, the temperature dependence of the viscosity is $\exp \left(E^{*} / R T\right)$, where $T$ is absolute temperature, $R=8.3 \mathrm{~J} \mathrm{~mol}^{-1} \mathrm{~K}^{-1}$, and a typical value of $E^{*}$ is 523 
kJ $\mathrm{mol}^{-1}$. Because of the high surface temperature of Venus $(750 \mathrm{~K})$, one might suppose that its lithosphere will be more deformable, but if we suppose the sublithospheric temperature is $T_{a}=1500 \mathrm{~K}$ and has viscosity $\eta_{a}$, then the effective surface viscosity would be $\eta_{s}=10^{18} \eta_{a}$. Of course, the rheology will be elasticplastic in the lithosphere; nevertheless this indicates that, in the absence of a specific subduction mechanism, mantle convection on Venus will be of the rigid lid type [Nataf and Richter, 1982; Solomatov and Moresi, this issue], as is well-known for variable viscosity fluid convection.

Turcotte [1993] has advanced a conceptual model based on rigid lid convection which is consistent with catastrophic global resurfacing. After a resurfacing event, the cold lithosphere has been swept away, and a new rigid lid grows conductively, with vigorous convection underneath. As this lid thickens, its negative buoyancy increases, until at some critical thickness, it is able to subduct, and thereby instigates a new resurfacing. In Turcotte's view, the incipient subduction zones at coronal margins may be the portents of a new resurfacing event.

In order to validate this point of view, it is necessary to have a mechanical understanding of how subduction is initiated in the rigid lid convective style of a variable viscosity fluid. The present paper develops'a criterion for transient subduction, based on a mechanism proposed by Fowler [1993]. The physics of this mechanism is explained in the following section.

\section{A Physical Model of Subduction}

The equations governing variable viscosity convection are complicated, and they have only recently been solved with parameter values approximately appropriate for planetary mantles [Moresi and Solomatov, 1995]. In particular, such numerical solutions as have been obtained do not reveal subduction zones, unless these are artificially implanted [Gurnis, 1989]. However, variable viscosity convection can be analyzed successfully by asymptotic methods [Fowler, 1985], and this reveals that very high lithospheric stresses, of the order of 1-10 kbar $\left(10^{2}-10^{3} \mathrm{MPa}\right)$ are generated, due to the large negative buoyancy in the stagnant lid. These stresses can be in excess of the yield strength of lithospheric rocks, and indeed observations of crustal deformation bear witness to the resulting plastic behavior. Fowler [1993] showed that in this case (i.e., where a yield strength is identified), the description of rigid lid variable viscosity is only slightly modified. Specifically, if we consider a two-dimensional (steady) convecting cell, with an upwelling at $x=0$ ( $x$ is the horizontal coordinate) and a top surface at $z=0$ ( $z$ is the depth coordinate), then a strongly variable viscosity fluid convects vigorously, at high Rayleigh number, below a virtually stagnant lid $z=s(x)$, in which heat transfer is conductive.

The analyses of Fowler [1985] and Moresi and Solomatov [1995] show that the highest stresses occur near the top of the lid (near $z=0$ ), and hence Fowler [1993] showed that if failure occurs, one can identify a plastic lid with base $z=q(x)$ in which the yield stress is equaled, and the effective viscosity adapts itself so that this can be so. The nature of the convection is not altered though, providing $q<s$, that is, if the plastic zone lies wholly within the stagnant lid, since then the effective plastic viscosity is still very large.

Now it is found that as $x$ increases, there is a point, $x=x_{F}$ say, at which $q$ reaches $s$. If $x_{F}$ lies within the width of the convection cell, then at that point, the effective plastic viscosity is equal to that of the underlying mantle. Thus near this point, the effective lithosphere viscosity is such that it can partake in the circulation. In effect, the lithosphere is broken.

This, then, is the proposed mechanism for subduction. Our purpose in this paper is to examine the model when the stagnant lid base $s$ and the plastic lid base $q$ depend on time. If transient subduction is to be viable in this model, then we require that there exists a failure time such that $q<s$ for $t<t_{F}$, and $q$ reaches $s$ at $x=x_{F}$ at time $t=t_{F}$. Additionally, we need the location $x_{F}$ and time $t_{F}$ for failure to make geophysical sense. To be consistent with Turcotte's [1993] concept of lithospheric failure outside coronas, we would like $x_{F} \sim 200 \mathrm{~km}$, and $t_{F} \sim 500 \mathrm{Ma}$, for example.

The analysis, which is briefly outlined in the appendix, is very complex, and so here we describe the form of the solution we find. After an overturning, stagnant lid convection resumes. The lid grows in thickness due to thermal conduction, but the convective flow below has a faster timescale and responds instantaneously to the changing lithosphere thickness. In particular, Fowler [1985] showed that the lithosphere thickness is entirely determined by the dynamics of a "delamination layer", more specifically a thin thermal boundary layer at the base of the lithosphere where the temperature gradient switches from conductive to adiabatic, and the strain rate increases from virtually zero toward the convective value below. We assume this is still true, and since this delamination layer timescale is very rapid, its dynamics are effectively time independent. We find that this gives a relation between the location $z=s$ of the stagnant lid base, and the heat flux at the base of the lithosphere. This extra condition, together with transient heat conduction in the lid and thermally prescribed surface and lid base temperatures, is sufficient to determine the time-evolving temperature and lithosphere thickness.

Having determined (at least in principle) the lid thickness and the lid temperature, the momentum equations now determine the lithospheric stresses. If these exceed the yield stress, then we posit a viscoplastic rheology such that if the second stress invariant $\tau$ reaches a critical value $\tau_{c}$, then a Von Mises yield criterion is adopted, wherein the viscosity $\eta$ becomes indeterminate, but is chosen so that $\tau=\tau_{c}$. This defines a plastic zone, whose thickness $q$ is determined by matching stress conditions from the plastic to the viscous part of the stagnant lid; there is in fact a boundary layer there, and the stresses jump rapidly. 
In our calculation below, we nondimensionalise and scale the equations. We have to solve a complicated free boundary problem for $s$, but it turns out that a similarity solution is appropriate, and the problem reduces to a relatively straightforward numerical computation. When this is solved, we then find that indeed $q<s$ (failure does not occur) for small times $t$, and there is a nonzero value $t_{F}$ when failure occurs, at a location $x=x_{F}$ from the upwelling. These results and their interpretation are discussed further in the final section of the paper.

\section{Mathematical Model}

Convection of a planetary mantle where subduction does not occur can be described by the boundary layer theory of Fowler [1985, 1986], which applies to the convection of fluids with strongly temperature dependent viscosity. The bulk of the temperature drop occurs across a thick, cold, stagnant lid, and the analysis shows that the negative buoyancy generated in this lid causes large stresses to exist. Accordingly, Fowler [1993] extended his previous analyses to allow for a plastic yielding within the lid, when the stress exceeds the yield stress, typically expected to be of the order of kilobars. (There is a mistake in that paper whose quantitative effect is quite small: it is rectified in the appendix.) In turn, this leads to a hypothesis for the onset of subduction when the plastic region extends to the base of the stagnant lid. When failure occurs, the situation is as depicted schematically in Figure 1. The effective viscosity of the plastic region is continuous with that beneath, so that when the plastic region extends to the base of the lid, the lid viscosity is low, comparable to that in the convecting layer beneath. Consequently, it will partake in the convection, and the cold lid will subduct.

The analysis which follows is included here for completeness, but is only described in outline, for two rea-

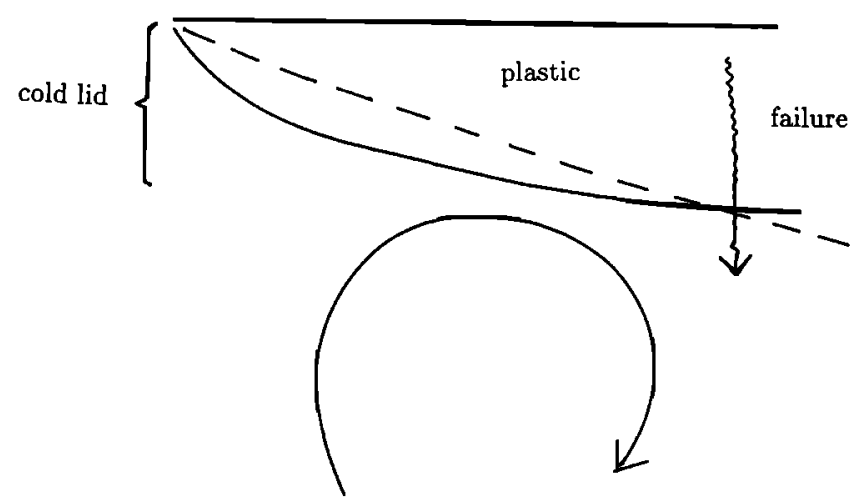

Figure 1. Cartoon of a convection cell underneath a stagnant lid. Within the lid, a plastic region exists, where the stress equals the yield stress. When this plastic region extends to the base of the stagnant lid, the effective lid viscosity at the point of intersection becomes equal to that of the underlying mantle, the lithosphere effectively fails, and subduction can occur. sons. The analysis is closely based on previous work by Fowler [1993] and differs only through the time dependence of the lithosphere temperature and of the plastic base $z=q(x, t)$ and lithosphere base $z=s(x, t)$. A more complete account will be published elsewhere and, in particular, will solve the three-dimensional (radially symmetric) problem, which has further complications which we do not dwell on here. In this section we describe the form of the solution in two dimensions, which will be necessary for comparison with any direct numerical computations which may be done in the future.

Let $(x, z)$ be horizontal and vertical (downward) Cartesian coordinates, with $x=0$ being the location of a mantle plume. This plume drives a horizontal flow in the lithosphere, equations for which have been analyzed by Fowler [1993]. We therefore limit ourselves to a description of the principal feature of the model and of where the analysis differs from the earlier one.

As is common in variable viscosity convection, the flow is divided into two main parts. There is a cold (lithospheric) lid in which the flow velocity is extremely small, so that the temperature field is described by thermal conduction. Below this lid, the mantle is approximately isoviscous and flow is rapid, characterized by a high Rayleigh number. We use nondimensional variables, as described by Fowler [1993]; specifically, we define a horizontal length scale $l$, a vertical lengthscale $d$, and a conductive time scale $d^{2} / \kappa$, where $l$ is taken as the mantle depth, $\kappa$ is the thermal diffusivity, and $d$ is defined by

$$
d=\nu\left(\frac{T_{a}-T_{s}}{T_{a}}\right)^{4 / 5} l
$$

where $T_{a}$ is asthenospheric temperature, $T_{s}$ is surface temperature, and $\nu$ is given by

$$
\nu=\frac{1}{\varepsilon}\left(\frac{\sigma}{R a}\right)^{1 / 5}
$$

the viscosity number $\varepsilon$ and the Rayleigh number $R a$ are defined by

$$
\varepsilon=\frac{R T_{a}}{E} \quad R a=\frac{\alpha \rho_{a} g l^{3} T_{a}}{\eta_{a} \kappa},
$$

where $R$ is the gas constant, $E$ is the activation energy for viscosity, $\alpha$ is the thermal expansion coefficient, $g$ is gravitational acceleration, and $\eta_{a}$ is asthenospheric viscosity. Typical values for the Earth are $\varepsilon=1 / 40$, $R a=4 \times 10^{7}$, for which $\nu \sim 1$, so $d \sim l$, and $d^{2} / \kappa \approx 10^{11}$ years. The parameter $\sigma$ is a measure of the strength of the plume which is supposed to impinge at $x=0$. In steady convection, $\sigma=1$, and the convective flow below the lid is driven by the upwelling buoyant jet at $x=0$. For the case of an isolated plume (typical for higher Rayleigh number convection), the plume head flows outwards under a pressure head beneath the lid of order $\alpha \rho_{a} g d_{p} \Delta T$, where $d_{p}$ is the plume diameter, and $\Delta T$ is its excess temperature over the ambient temperature $T_{a}$. It can be shown that this pressure head matches to the dynamically induced pressure below the 
lid (of the order of $\left.\left(\eta_{a} \kappa / l^{2}\right) R a \nu \varepsilon^{2}\right)$, providing $\nu$ is defined by

$$
\nu=\left(\frac{d_{p}}{l}\right)\left(\frac{\Delta T}{T_{a}}\right) \frac{1}{\varepsilon^{2}},
$$

and $\sigma$ is then given from (2) by

$$
\sigma=R a(\varepsilon \nu)^{5} .
$$

In reality, $d_{p}$ (and thus $\sigma$ ) will decrease as the plume spreads. If we choose $d_{p}=60 \mathrm{~km}, \Delta T=50 \mathrm{~K}, T_{a}=$ $1500 \mathrm{~K}, l=3000 \mathrm{~km}$, then for $\varepsilon=1 / 40, \nu \sim 1$, and less intense plumes give smaller values of $\nu$. In this paper we concentrate on the case $\sigma=1$, corresponding to quasi-steady convection.

Let us denote the location of the lid 'base' as $z=$ $s(x, t)$. In $0<z<s$, the normalised temperature problem is then

$$
\begin{gathered}
\theta_{t}=\theta_{z z} \quad 0<z<s \\
\theta=0 \quad z=0 \\
\theta=1, \quad \theta_{z}=\gamma \quad z=s
\end{gathered}
$$

where subscripts denote partial derivatives and $\theta$ is the scaled temperature. The condition on $z=0$ represents application of a prescribed surface temperature, while that at $z=s(\theta=1)$ represents prescription of an asthenospheric temperature. The lid base $z=s$ is an unknown and must be determined as part of the solution (this is therefore a free boundary problem). If $\gamma$ is known, then the extra flux condition at $z=0$ (analogous to a Stefan condition) enables $s$ to be found. However, determination of $\gamma$ requires further analysis.

\section{Uplift}

The buoyant uplift is determined just as was done by Fowler [1993] and is found to be (relative to an arbitrary level $z=0$ )

$$
h=-\alpha T_{a} \nu l\left(\frac{T_{a}-T_{s}}{T_{a}}\right)^{9 / 5} \int_{0}^{s}(1-\theta) d z .
$$

\section{Delamination layer}

At the base of the lid is a thin delamination layer through which the velocity begins to increase, and the temperature adapts to the constant subasthenospheric value. This layer is analyzed by Fowler [1993], and the same analysis applies here because this layer will relax rapidly to (quasi-) equilibrium. The result of that analysis yields a condition on $\gamma$, which is in the present context

$$
\frac{\sigma}{\gamma} \frac{\partial}{\partial x}\left[\frac{1}{\gamma^{3}} \frac{\partial s}{\partial x}\right]=A,
$$

where $A$ is a constant determined numerically as $A=$ 0.087: $\sigma$ is generally given by (4) and (5). By solving (6) together with (8), we can calculate both the temperature field $\theta$ and the lid base position $z=s(x, t)$. A prescription of appropriate boundary conditions for (8) is discussed below.

\section{Plastic lid}

The stresses in the cold lid become very large, and we propose a viscoplastic rheology to deal with this, using the Von Mises yield criterion. If the (dimensional) yield stress is $\tau_{c}$, then the plastic region is $0<z<q(x, t)$, in which the second stress invariant $\tau_{i j} \tau_{i j}=2 \tau_{c}^{2}$ (where $\tau_{i j}$ is the stress deviator tensor). An analysis identical to that of Fowler [1993] then shows that $q$ is given by

$$
q=\frac{6}{\lambda} \int_{0}^{s} z(1-\theta) d z
$$

where the dimensionless parameter $\lambda$ is given by

$$
\lambda=\frac{12 R T_{a}^{2} \tau_{c}}{E \eta_{a}^{1 / 5} \kappa^{1 / 5}\left(\alpha \rho_{a} g\right)^{4 / 5} l^{2 / 5}\left(T_{a}-T_{s}\right)^{9 / 5}} .
$$

The parameter $\lambda$ is a measure of the size of the yield stress; if $\lambda$ is small, then $q$ is large, and the whole lithosphere will be plastic (and would thus founder), whereas if $\lambda$ is large, plasticity is confined (if it occurs at all) to a thin skin near the top.

The size of this parameter is thus of some relevance. For the Earth, we estimate it using values $R=8.3$ $\mathrm{J} \mathrm{mol}^{-1} \mathrm{~K}^{-1}, T_{a}=1500 \mathrm{~K}, \tau_{c}=1 \mathrm{kbar}\left(10^{8} \mathrm{~Pa}\right)$, $E=125 \mathrm{kcal} \mathrm{mol}^{-1}\left(523 \mathrm{~kJ} \mathrm{~mol}^{-1}\right), \eta_{a}=10^{21} \mathrm{~Pa} \mathrm{~s}$, $\kappa=10^{-6} \mathrm{~m}^{2} \mathrm{~s}^{-1}, \alpha=3 \times 10^{-5} \mathrm{~K}^{-1}, \rho_{a}=3 \times 10^{3}$ $\mathrm{kg} \mathrm{m}^{-3}, g=10 \mathrm{~m} \mathrm{~s}^{-2}, l=3000 \mathrm{~km}, T_{s}=300 \mathrm{~K}$. We then have $\lambda \approx 0.34$. The fact that $\lambda$ turns out to be $O(1)$ is an indication of the viability of the mechanism discussed here. The precise value of $\lambda$ depends on the exact values used, but we would expect the value for Venus to be similar.

Fowler [1993] analyzed the steady solutions of this model (corresponding to steady convection), for which $\theta=z / s$ and thus $q=s^{2} / \lambda$. Failure occurs if $q$ reaches $s$, that is, if $s>\lambda$. As the lid thickness increases away from the upwelling, failure will occur if $\lambda$ is sufficiently small. Specifically, Fowler [1993] found $s \approx 0.82 x^{2 / 5}$ for steady convection, so that for a $3000 \mathrm{~km}$ wide convection cell, plastic failure leading to subduction and active plate tectonics could occur in this model if $\lambda<0.82$, approximately. This assumed $s(0)=0$, which may not be an appropriate assumption however. This is discussed further below.

\section{Time Dependent Results}

\section{Problem Formulation}

We investigate the situation following resumption of sublithospheric convection beneath the surface of a planetary mantle, following a resurfacing event. Two principal scenarios can be imagined. If the sublithospheric convection is essentially steady (cellular, rather than intermittent), then the "plume" parameter $\sigma=1$. However, if convection is intermittent, perhaps due to 
a higher Rayleigh number, then $\sigma$ decays with time, corresponding to the spread of a globular plume. In two dimensions, a plume has depth $d_{p}$ and width $w_{p}$ related by $d_{p} w_{p}=$ const, and if we assume the spreading rate $d w_{p} / d t$ is proportional to the excess pressure head $\alpha \rho_{a} g d \Delta T \propto d_{p}$, then we have $d_{p} \sim t^{-1 / 2}$, so that $\sigma \sim t^{-5 / 2}$. More generally, we might suppose that

$$
\sigma=\sigma_{0} t^{-\beta}
$$

Then a solution can be found in terms of similarity variables

$$
\xi=\frac{x}{2^{5 / 2} \sigma_{0}^{1 / 2} t^{(5-2 \beta) / 4}} \quad \eta=\frac{z}{2 t^{1 / 2}},
$$

and is given by

$$
s=2 t^{1 / 2} S(\xi) \quad \theta=g(\xi, \eta) \quad \gamma=G(\xi) /\left(2 t^{1 / 2}\right) .
$$

From (6) and (8), $g$ satisfies

$$
\begin{gathered}
-(5-2 \beta) g_{\xi}=g_{\eta \eta}+2 \eta g_{\eta} 0<\eta<S(\xi), \\
g=0 \quad \eta=0 \\
g=1, \quad g_{\eta}=G(\xi) \quad \eta=S(\xi),
\end{gathered}
$$

where $G$ is determined from

$$
\frac{1}{G} \frac{d}{d \xi}\left[\frac{1}{G^{3}} \frac{d S}{d \xi}\right]=A .
$$

We see that $\ln (1 / \xi)$ is a time-like variable (if $\beta<5 / 2$ ), since $\partial g / \partial(\ln \{1 / \xi\})=-\xi \partial g / \partial \xi$, and that $\xi \rightarrow 0$ (i.e., $x \rightarrow 0)$ corresponds to long "time", while $\xi \rightarrow \infty(x \rightarrow$ $\infty)$ corresponds to short time. Since the equation for $g$ is diffusive, we can expect an error function profile for large $\xi$. If we assume

$$
g \approx \operatorname{erf}(\eta) \xi \rightarrow \infty,
$$

then for consistency, $S$ is large; also

$$
G \approx \frac{2}{\sqrt{\pi}} e^{-S^{2}}
$$

so that (15) is approximately

$$
e^{S^{2}} \frac{d}{d \xi}\left[e^{3 S^{2}} \frac{d S}{d \xi}\right]=\frac{16 A}{\pi^{2}}
$$

By using Laplace's method for the asymptotic evaluation of integrals, we can then find the limiting form of $S$ as $\xi \rightarrow \infty$ as

$$
\exp \left(2 S^{2}\right) \sim\left[(128 A S)^{1 / 2}\left(\xi+\xi_{0}\right) / \pi\right]+\hat{c} S^{3}
$$

where $\xi_{0}$ and $\hat{c}$ are arbitrary constants.

On the other hand, as $\xi \rightarrow 0, g$ tends to the "steady" solution $g=\eta / S(\xi)$, whence $G \rightarrow 1 / S$, and so $S$ satisfies $S\left(S^{3} S^{\prime}\right)^{\prime}=A$, with solution satisfying $S(0)=S_{0}$, $S^{\prime}(0)=S_{0}^{\prime}$,

$$
\int_{S_{0}}^{S} \frac{S^{3} d S}{\left(S^{3}-c\right)^{1 / 2}}=\left(\frac{2 A}{3}\right)^{1 / 2} \xi
$$

where $c=S_{0}^{3}-3 S_{0}^{6} S_{0}^{\prime 2} / 2 A$. If $S_{0}=0$, (20) devolves to the similarity form $S=k \xi^{2 / 5}$ given by Fowler [1993].

\section{Numerical Solution}

In order to solve (14) and (15), we must pose boundary conditions (in $x$ ) for $S$. Equation (15) requires two conditions, and it is natural to pose both of these at $\xi=0$. One possibility is that $S \rightarrow 0$ as $\xi \rightarrow 0$. However, the correct limiting description of $S$ for small $\xi$ depends on an analysis of plume head conditions for small $x$, a task avoided by Fowler [1985]. Numerical computations by Moresi and Solomatov [1995] suggest that $S(0) \approx 0.45$ (see their Figure 4 ), and this may be appropriate when $\nu=O(1)$. Below we give results for both situations. (The extra condition of symmetry, that $S^{\prime}(0)=0$, is likely to be forced as a local correction due to plume head dynamics.)

Our strategy is to solve (14) and (15), with initial conditions for large $\xi$ given by (16) and (19). The values of $\xi_{0}$ and $\hat{c}$ are adjusted in order that $S(0)=S_{0}$, the prescribed value. The results of these computations are shown in Figures 2 and 3.

The base of the plastic zone is given from (9) by

$$
\begin{gathered}
q=2 \sqrt{t} Q \\
Q=\frac{12 \sqrt{t}}{\lambda} \int_{0}^{S} \eta(1-g) d \eta
\end{gathered}
$$

and it is clear that $Q<S$ for small $t$; hence $q<s$, and failure first occurs when $Q$ touches $S$ as shown in Figures 2 and 3. It is clear from these figures that it is difficult to identify an effectively precise location of failure. At a critical time given by $t=(\lambda \tau)^{2}$, where in Figure 2 (with $S(0)=0$ ) $\tau=0.58, Q$ reaches $S$ at a value $\xi=2.01$, where $S=1.29$. The corresponding values for $S(0)=0.45$ are $\tau=0.58, \xi=5.4, S=$ 1.29. Essentially, at this critical time, the lithosphere becomes fluid, and subduction would be expected to occur where the lithosphere founders. This theory is not able to be specific as to the length scale over which it takes place, however.

\section{Discussion}

The theory of the preceding section predicts that in a convecting variable viscosity mantle, lithospheric failure will occur following a resurfacing at a dimensional time $t_{F}$ given by

$$
t_{F} \approx \frac{d^{2} \lambda^{2} \tau^{2}}{\kappa},
$$

where $S \approx 1.3$. This scenario assumes that following a resurfacing event, quasi-steady sublithospheric convection resumes below a growing stagnant lid. The crucial depth scale is $\delta=\lambda d$, in terms of which 


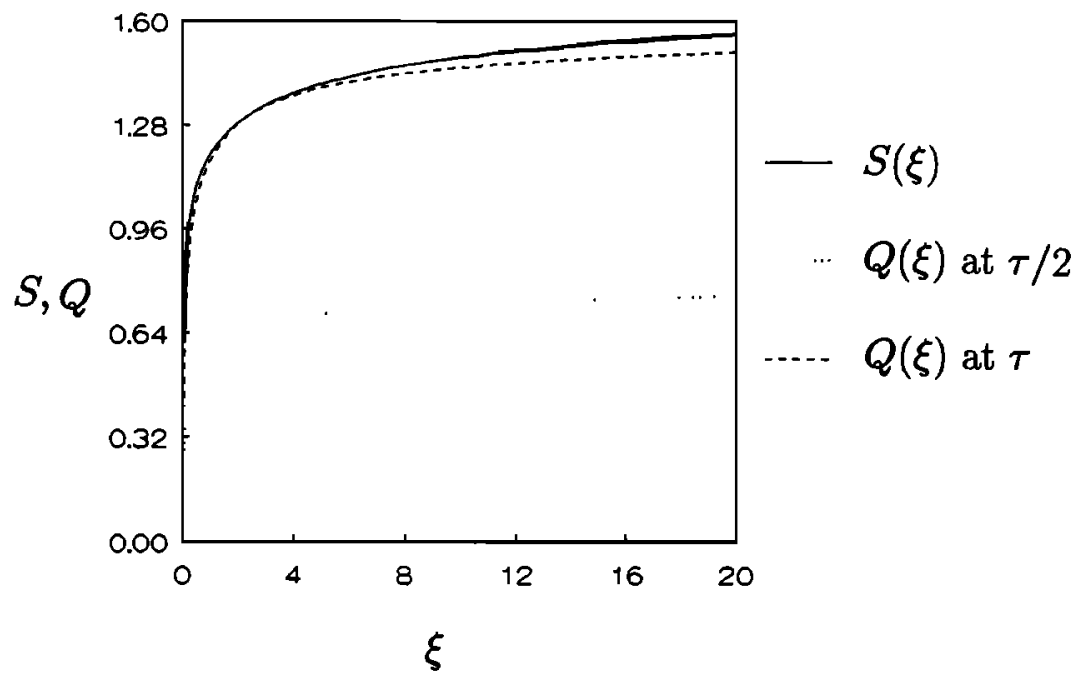

Figure 2. Computation of the plastic lid base $z=q$ and the stagnant lid base $z=s$. The results are plotted in the similarity forms $\eta=Q$ and $\eta=S$. In these coordinates, $S(\xi)$ is fixed and given by the solid curve for the case $S(0)=0$. The plastic base $Q$ is proportional to $t^{1 / 2}$ and is shown at the critical time $t^{1 / 2} / \lambda=\tau=0.58$ (dashed line), and when $t^{1 / 2} / \lambda=\tau / 2$ (dotted line). It can be seen that failure occurs virtually synchronously along the lid.

$$
t_{F} \approx 0.34 \delta^{2} / \kappa \quad z_{F} \approx 1.5 \delta .
$$

The depth $\delta$ is defined, via (1) and (10), as

$$
\delta=\frac{12 \tau_{c}}{\alpha \rho_{a} g\left(T_{a}-T_{s}\right)}
$$

and this is the critical depth of interest. For the Earth, with $\tau_{c}=1 \mathrm{kbar}, T_{a}=1500 \mathrm{~K}, T_{s}=300 \mathrm{~K}$, and other parameters as before, we have $\delta \approx 1100 \mathrm{~km}$, thus $z_{f} \approx 1600 \mathrm{~km}, t_{F} \approx 10^{4}$ m.y.

Let us first explain our results for an ideal mantle having a temperature dependent viscoplastic rheology of the type we have described. The basic type of (quasisteady) convection is the rigid lid type, having a lid of thickness $\approx d$ given by $(1)$. Strictly, this theory assumes $d \ll l$, although for the Earth it seems $d \sim l$. For such a steady convective state, the lid will fail plastically via subduction if the parameter $\lambda$ given by (10) is less than some critical value of the order of 1 . Fowler [1993] suggested this was if $\lambda<0.82$, but whatever the precise value, what is of interest is that a typical value for the Earth (and presumably also Venus) is $\lambda \approx 0.34$. This suggests that this failure mechanism is viable for the Earth and Venus.

The issue in this paper is then whether such a failure can occur in a semiperiodic manner. Following a resurfacing event, the rigid lid grows conductively toward its equilibrium thickness $\sim d$. It is only if $\lambda \ll 1$,

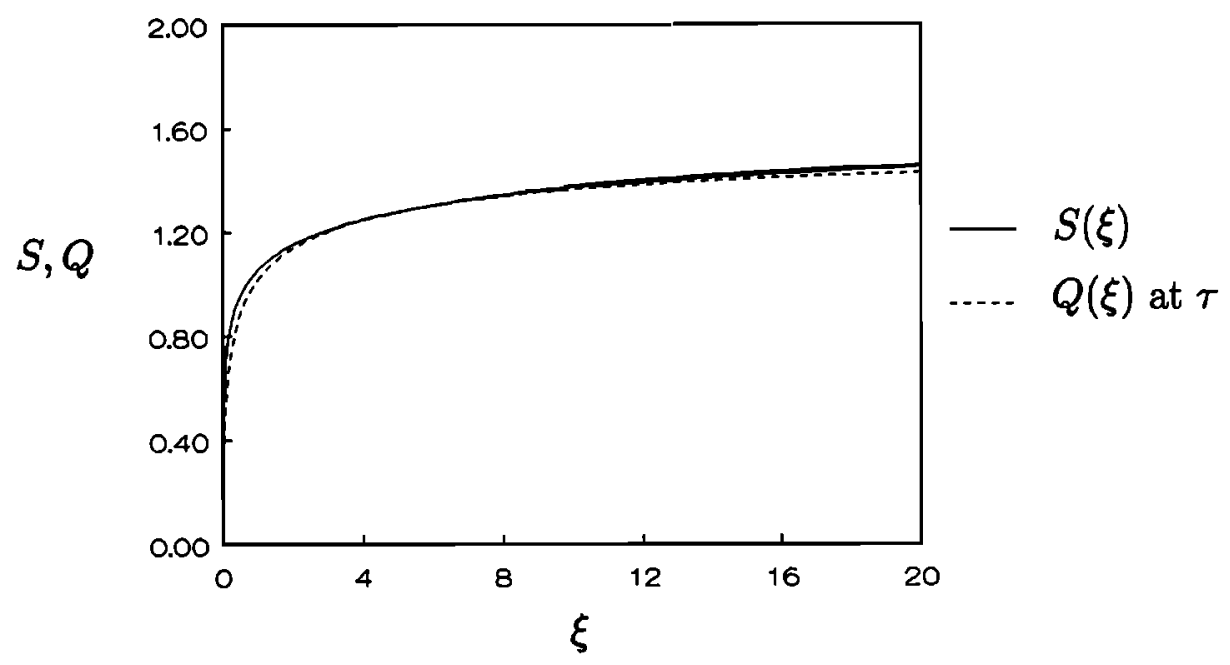

Figure 3. The same computation as for Figure 2, but with $\xi_{0}$ and $\hat{c}$ chosen so that $S(0)=0.45$.

Failure occurs at the same value of $t^{1 / 2} / \lambda=0.58$ along the length of the lid. 


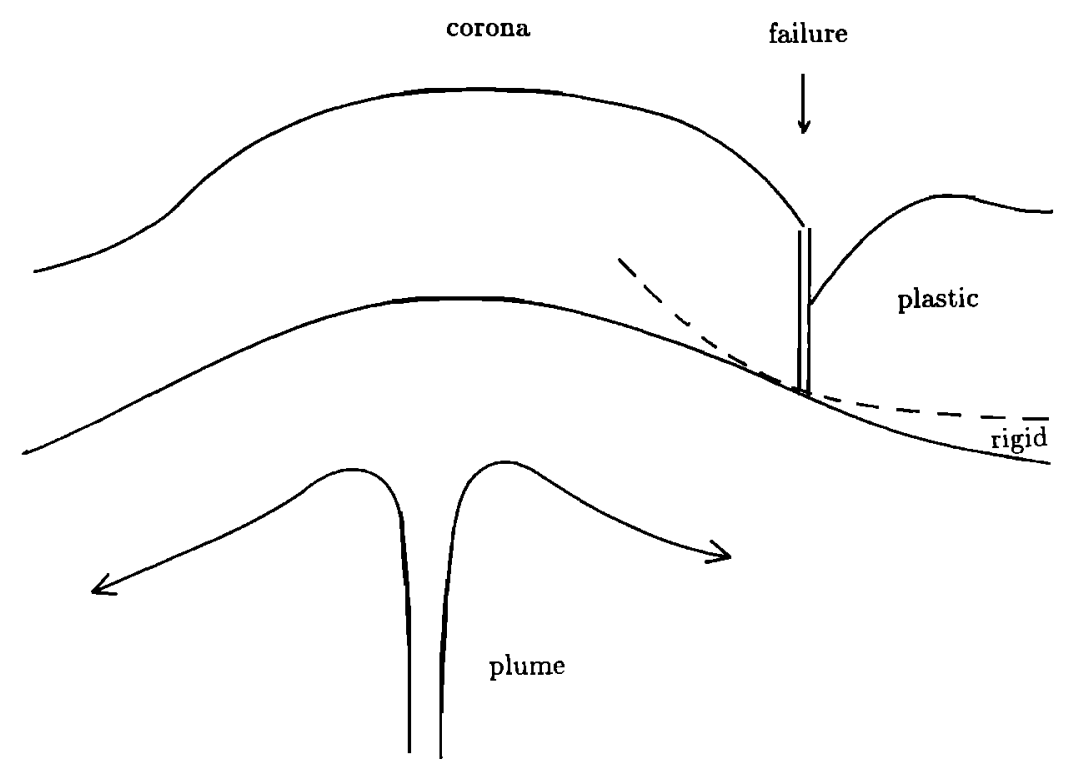

Figure 4. A schematic interpretation of the results for tectonics on Venus. A mantle plume causes a coronal uplift to develop, and as the lithosphere thickens, failure occurs in the stagnation point flow away from the coronal center, at distance $\sim x_{F}$ and time $t_{F}$. The failure effectively breaks the lithosphere, allowing it to founder. The asymmetry caused by the underlying shear flow causes the outer rim to bend downward since the plume buoyancy tends to support the inner part of the corona.

i.e., $\delta \ll d$, that a subsequent failure will occur while the lid is relatively thin. Although there are some reasons (discussed below) why the effective value of $\lambda$ may be lower than the value given here, the import of the present calculation as it stands is that (1) Earth's mantle (and presumably that of Venus) is likely to founder, but that (2) it becomes quite thick before doing so (and hence also the interval between resurfacing is very long, of the order of $10^{4} \mathrm{~m} . \mathrm{y}$.). This mechanism is therefore best considered marginal, but we now point out some ways in which the quantitative details may be modified.

First, the yield strength will vary with depth and might be expected to be lower at higher temperatures. Possibly more important to the heat budget problem is the presence of radioactive heating, which will have a significant effect on the lid thickness evolution. An inclusion of this term would be of some interest. Fowler [1993] argued that radioactive heating could cause a significantly thinner rigid lid.

Supposing the present mechanism is viable, why should Earth have quasi-steady cellular suboceanic convection, while Venus may have episodic convection? This could be due to a higher value of $\lambda$ on Venus than on Earth. If failure occurs when the lithosphere is relatively thin (small $\lambda$, or higher internal heating), then the negative buoyancy associated with overturn is less, and might be expected to lead to quasi-steady convection. For larger $\lambda$ (or smaller heating), the lid is thicker at failure, and the resulting convective episode can be expected to be catastrophic, much as in Howard's [1966] model at higher $R a$. In summary, we consider the present mechanism to be potentially viable to explain tectonic styles of both Venus and Earth, but it will require further work to be more specifically predictive.
There are two obvious limitations to our analysis. First, we have analyzed a Cartesian cellular geometry. We consider this to be a qualitative constraint which will not alter the basic physics of the problem. A second, possibly more serious, concern is the choice of a viscoplastic rheology. The rheology of the lithosphere varies from elastic to elastic-plastic to viscous as temperature increases. Our neglect of the elastic part of the rheology is motivated certainly by the wish to solve an "easier" problem, but also because viscoelastic fluids undergoing finite shear strain do in fact behave like (nonlinear) viscous fluids [e.g., Bird et al., 1977], and we do not see any reason to suppose that inclusion of elasticity will affect our results. Nevertheless, we do intend to include elastic effects in future studies.

Finally, how do we wish to interpret the present theory in terms of Venusian tectonics? Our cartoon of events is illustrated in Figure 4, which indicates a plume (hot spot) flow beneath a corona. Failure occurs at a distance away from the upwelling, and the asymmetry of the plume flow is then associated with the asymmetry of the resulting subduction. Lithospheric failure is associated with low values of $\lambda$, and for a given planetary mantle, $\eta_{a}$ increases as the planet cools; thus the tectonic evolution would be a competition between the decreasing $\lambda$, which would tend to promote failure, and the decreasing Rayleigh number, which decreases the effectiveness of convection.

Acknowledgments. Our thanks are due to Don Turcotte for his assistance, and to Slava Solomatov for sending us his papers prior to publication. S.B.G.O'B. acknowledges the support of a Royal Irish Academy se- 
nior visiting fellowship. This paper is dedicated to the memory of Alan Tayler.

\section{Appendix}

There is a mistake in the paper by Fowler [1993], which in fact renders the solution easier. First, the choice of $U$ scale in (12) (all equation numbers here refer to Fowler [1993]) should be

$$
U=\left(R a^{2 / 5} / \varepsilon\right) U_{0} .
$$

Now consider the rescaling of (17) in the plastic lid under (19). Evidently, (20c) is

$$
T_{1}^{2}+\nu^{2} \tau_{2}^{2}=c^{\prime 2},
$$

where

$$
c^{\prime}=\nu c .
$$

Thus (23) is replaced by

$$
T_{1}=-c^{\prime} \cos (\nu \phi) \quad \nu \tau_{2}=c^{\prime} \sin (\nu \phi),
$$

$\phi \rightarrow \nu \phi$ and $c \rightarrow c^{\prime}$ in (24), and the characteristic equations (25) are

$$
\begin{aligned}
\dot{x} & =-2 \nu \sin \nu \phi, \\
\dot{z} & =\cos \nu \phi, \\
\dot{\phi} & =-\frac{1}{c^{\prime}}\left[\Delta^{\prime}+\frac{\left(1-T_{0}\right)}{2} \frac{s^{\prime}}{s^{2}} z^{2}\right],
\end{aligned}
$$

together with $\phi=0$ on $z=0$. Evidently, for $\nu \ll 1$,

$$
\phi \sim \frac{\left(1-T_{0}\right)}{2 c^{\prime}} s s^{\prime}\left[\frac{z}{s}-\frac{z^{3}}{3 s^{2}}\right],
$$

using the definition of $\Delta$ in (31). The plastic viscosity is then

$$
\eta=\frac{-c^{\prime} \cos \nu \phi}{2 \nu^{2} \varepsilon^{2} \Lambda\left(u / \eta_{q}\right)^{\prime}} .
$$

The equations in the stress boundary layer are the same, and it follows from (42) that (at $z=q$ )

$$
q^{\prime}=\frac{\tau_{2}^{\text {plas }}-\tau_{2}^{\text {visc }}}{-2 T_{1}^{\text {plas }}}
$$

Since $T_{1}^{\text {plas }} \sim-c^{\prime}, \tau_{2}^{\text {plas }} \sim c^{\prime} \phi_{q}$, we find

$$
q^{\prime} \sim \frac{\left(1-T_{0}\right) s s^{\prime}}{6 c^{\prime}},
$$

whence

$$
q \sim \frac{\left(1-T_{0}\right)^{2} s^{2}}{12 c^{\prime}} .
$$

Notice that this is simply (49), replacing $c$ by $c^{\prime}$. A numerical solution for $q$ is not necessary, and in the subsequent discussion, $c^{*}$ can be taken as 0.046 , and $\tau_{c}$ can be replaced by $\nu \tau_{c}=\tau_{c} / \varepsilon R a^{1 / 5}$. For $\varepsilon=0.024$ and $R a \sim 3 \times 10^{7}, \nu \approx 1.2$, while for $R a \sim 3 \times 10^{9}, \nu \approx 0.5$.

\section{References}

Arkani-Hamed, J., G.G. Schaber, and R.G. Strom, Constraints on the thermal evolution of Venus inferred from Magellan data, J. Geophys. Res., 98, 5309-5315, 1993.

Bird, R.B., R.C. Armstrong, and O. Hassager, Dynamics of Polymeric Liquids, vol. 1, John Wiley, New York, 1977.

Fowler, A.C., Fast thermoviscous convection, Stud. Appl. Math., 72, 189-219, 1985.

Fowler, A.C., Thermal runaway in the Earth's mantle, Stud. Appl. Math., 74, 1-34, 1986.

Fowler, A.C., Boundary layer theory and subduction, $J$. Geophys. Res., 98, 21,992-22,005, 1993.

Gurnis, M., A reassessment of the heat transport by variable viscosity convection with plates and lids, Geophys. Res. Lett., 16, 179-182, 1989.

Herrick, D.L., and E.M. Parmentier, Episodic large-scale overturn of two-layer mantles in terretrial planets, J. Geophys. Res., 99, 2053-2062, 1994.

Howard, L.N., Convection at high Rayleigh number, in Proceedinhgs, 11th International Congress on Applied Mechanics, edited by H. Görtler, pp. 1109-1115, SpringerVerlag, New York, 1966.

Kiefer, W.S., and B.H. Hager, A mantle plume model for the equatorial highlands of Venus, J. Geophys. Res., 96, 20,947-20,966, 1991.

Kirby, S.H., Rheology of the lithosphere, Rev. Geophys., 21, 1458-1487, 1983.

Koch, D.M., A spreading drop model for plumes on Venus, J. Geophys. Res., 99, 2035-2052, 1994.

Lenardic, A., W.M. Kaula, and D.L. Bindschadler, A mechanism for crustal recycling on Venus, J. Geophys. Res., 98, 18,697-18,705, 1993.

McKenzie, D., P.G. Ford, C. Johnson, B. Parsons, D. Sandwell, S. Saunders, and S.C. Solomon, Features on Venus generated by plate tectonic boundary processes, J. Geophys. Res., 97, 13,533-13,544, 1992.

Moresi, L.-N., and V.S. Solomatov, Numerical investigation of $2 \mathrm{D}$ convection with extremely large viscosity variations, Phys. Fluids, 7, 2154-2162, 1995.

Nataf, H.-C., and F.M. Richter, Convection experiments in fluids with highly temperature-dependent viscosity and the thermal evolution of the planets, Phys. Earth Planet. Inter., 29, 320-329, 1982.

Parmentier, E.M., and P.C. Hess, Chemical differentiation of a convecting planetary interior: consequences for a one plate planet such as Venus, Geophys. Res. Lett., 19, 20152018, 1992.

Phillips, R.J., R.F. Rambertas, R.E. Arvidson, I.C. Sarkar, R.R. Herrick, N. Izenberg, and R.E. Grimm, Impact craters and Venus resurfacing history, J. Geophys. Res., 97, 15,923-15,948, 1992.

Sandwell, D.T., and G. Schubert, Evidence for retrograde lithospheric subduction on Venus, Science, 257, 766-770, 1992.

Schaber, G.G., R.G. Strom, H.J. Moore, L.A. Soderblom, R.L. Kirk, D.J. Chadwick, D.D. Dawson, L.R. Gaddis, J.M. Boyce, and J. Russell, Geology and distribution of impact craters on Venus: what are they telling us?, $J$. Geophys. Res., 97, 13,257-13,301, 1992.

Solomatov, V.S., and L.-N. Moresi, Stagnant lid convection on Venus, J. Geophys. Res., this issue.

Solomon, S.C., The geophysics of Venus, Phys. Today, July 1993 issue, pp. 48-55, 1993.

Solomon, S.C., and J.W. Head, Fundamental issues in the geology and geophysics of Venus, Science, 252, 252-260, 1991.

Solomon, S.C., et al., Venus tectonics: an overview of Mag- 
ellan observations, J. Geophys. Res., 97, 13,199-13,255, 1992.

Steinbach, V., and D.A. Yuen, The effects of multiple phase transitions on Venusian mantle convection, Geophys. Res. Lett., 19, 2243-2246, 1992.

Strom, R.G., G.G. Schaber, and D.D. Dawson, The global resurfacing of Venus, J. Geophys. Res., 99, 10,899-10,926, 1994.

Turcotte, D.L., An episodic hypothesis for Venusian tectonics, J. Geophys. Res., 98, 17,061-17,068, 1993.
A.C. Fowler, Mathematical Institute, Oxford University, 24-29 St. Giles', Oxford OX1 3LB, England. (e-mail: Fowler@maths.ox.ac.uk)

S.B.G. O'Brien, Department of Mathematics, University of Limerick, Limerick, Republic of Ireland. (e-mail: Stephen.Obrien@ul.ie)

(Received May 9, 1995; revised October 19, 1995; accepted October 20, 1995.) 